\title{
Pulsations of rapidly rotating stars with compositional discontinuities
}

\author{
Daniel R. Reese ${ }^{1}$, Francisco Espinosa Lara ${ }^{2,3}$, Michel Rieutord ${ }^{2,3}$ \\ ${ }^{1}$ Institut d'Astrophysique et Géophysique de l'Université de Liège, \\ Allée du 6 Août 17, 4000 Liège, Belgium \\ email: daniel.reese@ulg.ac.be \\ ${ }^{2}$ Universté de Toulouse, UPS-OMP, IRAP, Toulouse, France \\ ${ }^{3}$ CNRS, IRAP, 14 avenue Edouard Belin, 31400 Toulouse, France
}

\begin{abstract}
Recent observations of rapidly rotating stars have revealed the presence of regular patterns in their pulsation spectra. This has raised the question as to their physical origin, and, in particular, whether they can be explained by an asymptotic frequency formula for low-degree acoustic modes, as recently discovered through numerical calculations and theoretical considerations. In this context, a key question is whether compositional/density gradients can adversely affect such patterns to the point of hindering their identification. To answer this question, we calculate frequency spectra using two-dimensional ESTER stellar models. These models use a multi-domain spectral approach, allowing us to easily insert a compositional discontinuity while retaining a high numerical accuracy. We analyse the effects of such discontinuities on both the frequencies and eigenfunctions of pulsation modes in the asymptotic regime. We find that although there is more scatter around the asymptotic frequency formula, the semi-large frequency separation can still be clearly identified in a spectrum of low-degree acoustic modes.
\end{abstract}

Keywords. stars: oscillations, stars: rotation, stars: interiors

\section{Introduction}

Recent observations of pulsation spectra in rapidly rotating stars have revealed the presence of frequency patterns. For instance, García Hernández et al. (2009, 2013) found recurrent frequency spacings in two $\delta$ Scuti stars observed by CoRoT, thereby allowing the construction of an echelle diagram in the latter case. Similarly, Breger et al. (2012, 2013) found multiple sequences of very uniformly spaced frequencies in a $\delta$ Scuti star observed by Kepler. These observations show that although the pulsation spectra of $\delta$ Scuti stars lack the simple frequency patterns present in solar-type pulsators, regular patterns do exist in such stars and need to be explained.

Among the various possible explanations, one particularly interesting option is the asymptotic frequency pattern for low-degree acoustic modes (i.e. island modes) in rapidly rotating stars, recently discovered through numerical (Lignières et al. 2006; Reese et al. 2008, 2009) and theoretical considerations (Lignières \& Georgeot 2008, 2009; Pasek et al. 2011,2012 ). Identifying such a pattern in rapidly rotating stars could yield useful information such as the mean density (Reese et al. 2008; García Hernández et al. 2013). However, an open question is to what extent the pattern is affected by strong gradients or glitches (such as $\mu$ gradients, ionisation zones, or boundaries of convective regions), and whether this can hinder its identification. In order to answer this question, we investigate the pulsation spectra of rapidly rotating models with sharp discontinuities. 

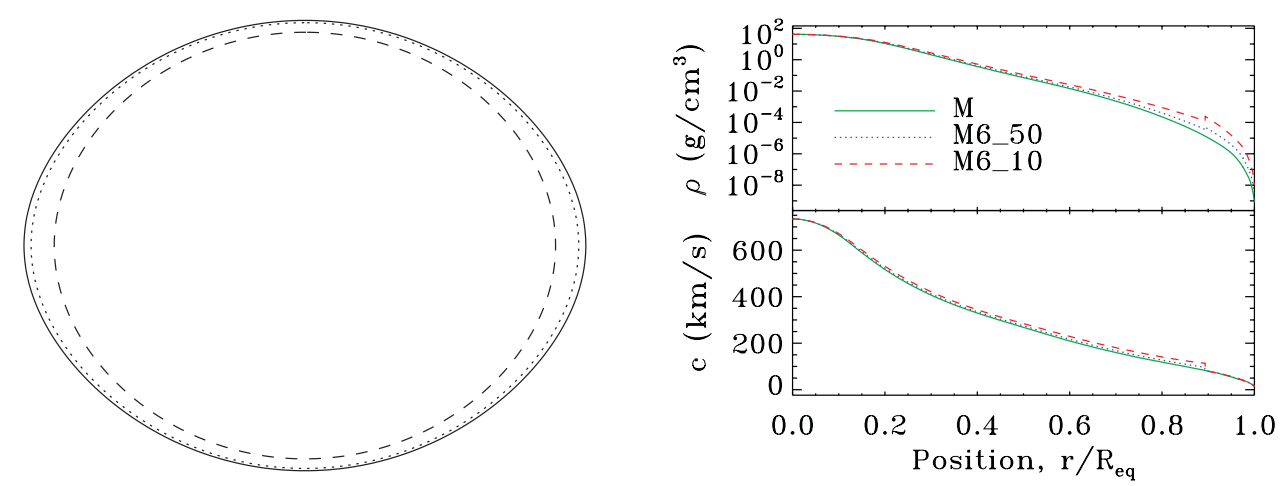

Figure 1. Left: Meridional cross-sections of discontinuities in models M6_xx (dashed line) and M7_xx (dotted line), and stellar surface (solid line). Right: Density and sound velocity profiles for three of the models.

\section{Numerical calculations}

We worked with various $3 M_{\odot}$ models, where the surface is rotating at $70 \%$ of the Keplerian break-up rotation rate $\left(v_{\text {eq }}=340-350 \mathrm{~km} \mathrm{~s}^{-1}\right)$. These models were produced by the 2D multi-domain spectral code ESTER, which self-consistently calculates the rotation profile, $\Omega$ (Rieutord \& Espinosa Lara 2009, 2013; Espinosa Lara \& Rieutord 2013). Its multi-domain approach is well-suited to introducing discontinuities without sacrificing numerical accuracy, since these can be made to coincide with domain boundaries. In what follows, we worked with five different models: M which is smooth, M6_50, M6_10, M7_50, and M7_10. Models M6_xx have a discontinuity deeper within the star (see Fig. 1, left panel). In all cases, the discontinuities follow isobars. The surface hydrogen content is decreased by $50 \%$ and $90 \%$ in models Md_50 and Md_10, and corresponds to a $17 \%$ and $39 \%$ jump in the speed of sound, respectively (see Fig. 1, right panel).

Adiabatic calculations of acoustic pulsation modes were carried out thanks to the TOP code which fully takes into account the effects of rotation (Reese et al. 2009). Regularity conditions were applied in the centre, the simple mechanical condition $\delta p=0$ was enforced at the stellar surface, and the perturbation to the gravity potential was made to vanish at infinity. Various matching conditions were needed to ensure that the perturbation of the pressure, the gravity potential, and its gradient, remain continuous across the perturbed discontinuity. Furthermore, the fluid domain had to be kept continuous by making sure that the deformation caused by the fluid displacement is the same below and above the discontinuity. Similar calculations had previously been carried out in Reese et al. (2011). However, these calculations did not take into account the fact that the matching conditions apply across the perturbed discontinuity, and the results were less conclusive because the discontinuity was located deeper within the star, where acoustic island modes are less sensitive.

\section{Results}

We first turn our attention to the effects of discontinuities on the eigenfunctions. Figure 2 shows the meridional cross-section of an island mode as well as the sound velocity and mode profile along a heuristically determined path. As can be seen in the right panel, the discontinuity modifies the wavelength as well as the amplitude of the oscillations. Further tests confirm that the wavelength scales with the sound velocity. 
Another effect which has already been pointed out by Reese et al. (2011) is a slight deviation of the mode at the discontinuity.
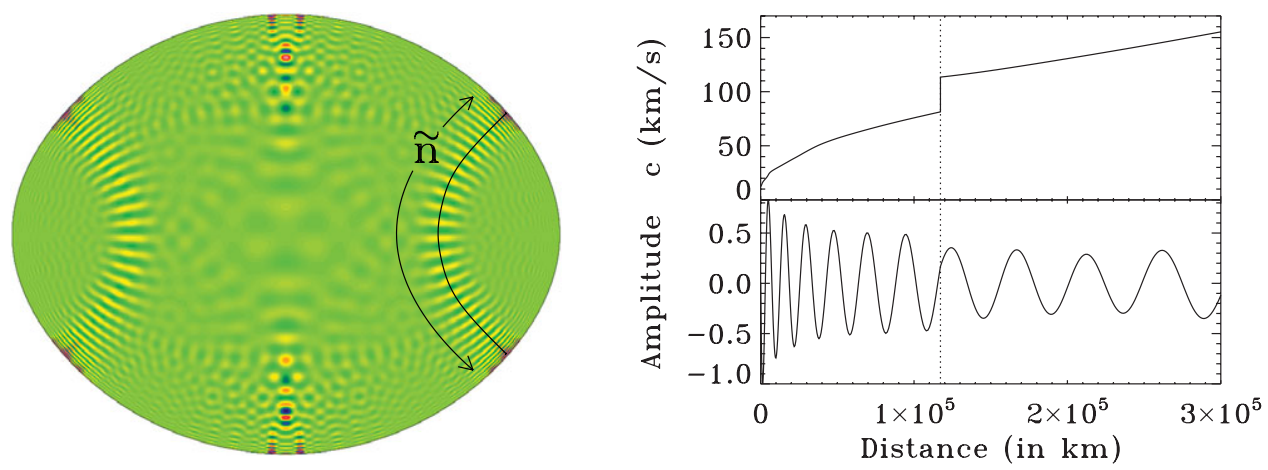

Figure 2. Left: Meridional cross-section of an island mode. Right: Sound velocity and mode profile along the path shown in the left panel (only part of the profile is shown for legibility).

At low rotation rates, discontinuities affect the frequencies by superimposing an oscillatory pattern over the usual frequency spectrum (e.g. Monteiro et al. 1994). A similar effect takes place here, as illustrated by the semi-large frequency separations shown in Fig. 3, although the oscillatory pattern is less regular. One can also calculate the scatter between the numerical frequencies and a simplified version of the asymptotic formula (see Eq. (27) of Reese et al. 2009). The scatter, $\left\langle\left(\nu_{\text {asymp. }}-\nu\right)^{2}\right\rangle^{1 / 2} / \Delta_{\tilde{n}}$, ranges from 0.0143 for model M to 0.0436 for model M7 10. Even in the best case, the scatter is more than an order of magnitude larger than the scatter obtained around the main sequence of equidistant frequencies found in Breger et al. (2012), thereby supporting the conclusion that this sequence is not caused by an asymptotic behaviour.

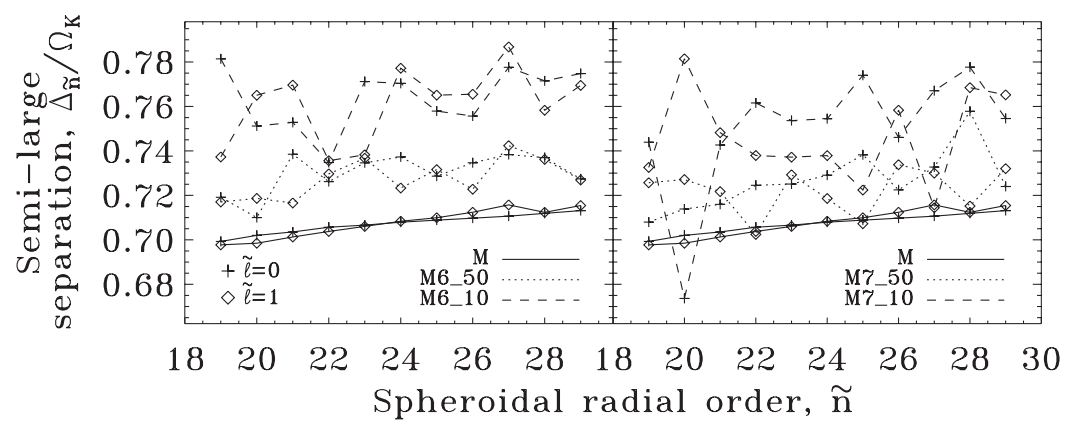

Figure 3. Semi-large frequency separation, $\Delta_{\tilde{n}}=\nu_{\tilde{n}+1}-\nu_{\tilde{n}}$, for axisymmetric $(m=0)$ modes, as a function of $\tilde{n}$, the spheroidal radial order (see Fig. 2, left panel).

One can then investigate whether it is possible to recover the semi-large frequency separation, $\Delta_{\tilde{n}}$. Figure 4 shows histograms of frequencies differences for three models. The lightly shaded areas show all frequency differences, whereas the dark areas show the frequency differences from modes with adjacent $\tilde{n}$ values and the same $(\tilde{l}, m)$ values. The upper row is based on the original numerical frequencies. In all cases, the semi-large frequency separation $\Delta_{\tilde{n}}$ shows up clearly. However, it turns out that the rotation rate is close to $\Delta_{\tilde{n}}$ thereby amplifying the signal, due to island mode multiplets (Pasek et al. 2012). This can be seen by comparing the light and dark regions in the histograms. In 


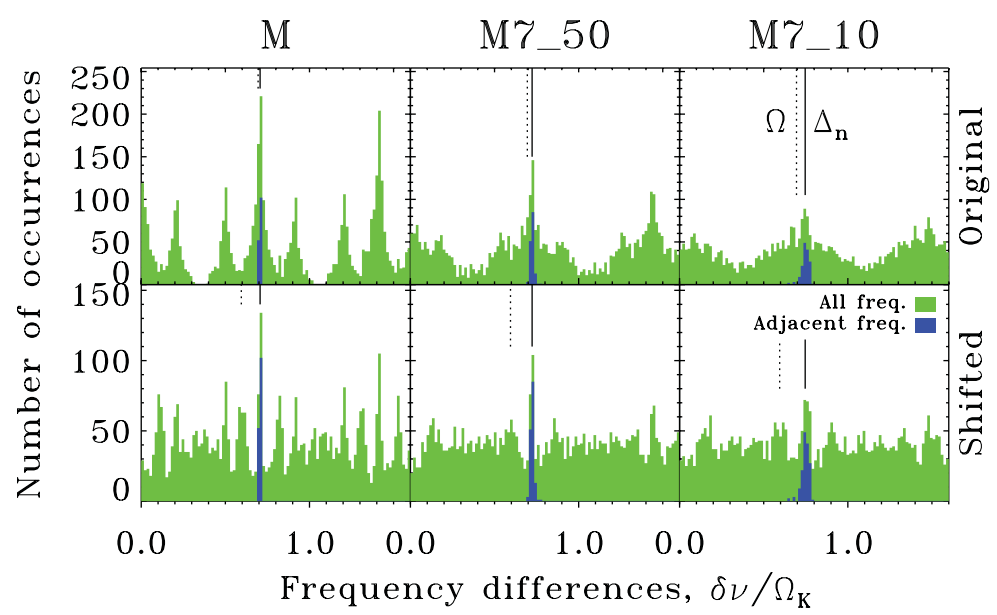

Figure 4. Histograms of frequency differences for three models (see text for details).

the lower row, the frequencies were shifted by $0.1 \mathrm{~m}$, thereby mimicking a lower rotation rate. Even in this situation, a peak remains at $\Delta_{\tilde{n}}$ for all three models.

In conclusion, although discontinuities lead to more scatter around the asymptotic behaviour of island modes and may complicate mode identification, they are unable to mask features such as the semi-large frequency separation. Hence, the asymptotic formula remains a viable explanation for stars such as those observed by García Hernández et al. (2009, 2013).

\section{Acknowledgements}

DRR is financially supported through a postdoctoral fellowship from the "Subside fédéral pour la recherche 2012", University of Liège. FEL and MR acknowledge the support of the French Agence Nationale de la Recherche (ANR), under grant ESTER (ANR-09-BLAN-0140).

\section{References}

Breger, M., Fossati, L., Balona, L. A., et al. 2012, ApJ, 759, 62

Breger, M., Lenz, P., \& Pamyatnykh, A. A. 2013, ApJ, 773, 56

Espinosa Lara, F. \& Rieutord, M. 2013, A $3 A$, 552, A35

García Hernández, A., Moya, A., Michel, E., et al. 2009, A $๒ A$, 506, 79

García Hernández, A., Moya, A., Michel, E., et al. 2013, A\&A, 559, A63

Lignières, F. \& Georgeot, B. 2008, Phys. Rev. E, 78, 016215

Lignières, F. \& Georgeot, B. 2009, $A \& A, 500,1173$

Lignières, F., Rieutord, M., \& Reese, D. 2006, A\&A, 455, 607

Monteiro, M. J. P. F. G., Christensen-Dalsgaard, J., \& Thompson, M. J. 1994, A\&A, 283, 247

Pasek, M., Georgeot, B., Lignières, F., \& Reese, D. R. 2011, Phys. Rev. Letters, 107, 121101

Pasek, M., Lignières, F., Georgeot, B., \& Reese, D. R. 2012, A\&̋A, 546, A11

Reese, D. R., Lignières, F., \& Rieutord, M. 2008, A\&A, 481, 449

Reese, D. R., MacGregor, K. B., Jackson, S., Skumanich, A., \& Metcalfe, T. S. 2009, A\& A, 506, 189

Reese, D. R., Espinosa Lara, F., \& Rieutord, M. 2011, in: C. Neiner, G. Wade, G. Meynet, \& G. Peters (eds.), Active OB stars: structure, evolution, mass loss, and critical limits, Proc. IAU Symposium No. 272 (Cambridge: Cambridge University Press), p. 535

Rieutord, M. \& Espinosa Lara, F. 2009, CoAst, 158, 99

Rieutord, M. \& Espinosa Lara, F. 2013, Lecture Notes in Physics, 865, 49 